\title{
EXPLORANDO CREENCIAS ACERCA DE LA NATURALEZA DE LA ENSEÑANZA EN MAESTROS DE CIENCIAS NATURALES DEL GRADO SEXTO EN BOGOTA, D. 0.
}

\author{
Lilia Reyes Herrera*, Gerardo Andrés Perafán Echeverri *, \\ Luis Enrique Salcedo Torres
}

\begin{abstract}
The exploration of science teachers' beliefs is one of the important topics of research for the science education community not only at the international but also at the national level.

The National policy about curriculum improvement reforms claims for the inclusion of inquiry practices in the educational process. The main research question is: What are teachers' beliefs about the nature of teaching? The research methodology used to approach this problem was qualitative. Transcripts from video cassettes were the main source of data.

Teachers believe that "things must be as they really are" and that "students must pay to the teacher what they owe" lo academic terms, jo order to achieve knowledge. These beliefs have important implications for the teaching of science. If we want teachers to change, we need to effectively support teachers' own beliefs inquiry.
\end{abstract}

\section{RESUMEN}

La exploración de las creencias de los maestros de ciencias es uno de los tópicos de investigación más importantes dentro de la comunidad de la educación en ciencias, no sólo a nivel nacional sino también internacional. Las políticas nacionales acerca de las reformas curriculares reclaman la inclusión de prácticas investigativas en los procesos educativos. La principal pregunta de investigación es: ¿Cuáles son las creencias de los maestros acerca de la naturaleza de la enseñanza? La metodología empleada para abordar este problema fue de tipo cualitativo. Las principales fuentes de datos fueron transcripciones de videos tomados en el aula de clase. Los maestros participantes en esta investigación creen que las "cosas deben ser tal como son" y que "los estudiantes deben pagar al maestro lo que le deben" en términos académicos. Estas creencias tienen implicaciones bien importantes para la enseñanza de las ciencias. Si queremos que los maestros cambien necesitamos apoyarlos en la investigación de sus propias creencias.

Instituciones participantes: Colegio Simón Rodríguez, Jornadas mañana y tarde; Colegio Cristóbal Colón, Jornada mañana; Liceo Femenino Antonia Santos, Jornada tarde.

PALABRAS CLAVE: Creencias, maestros, enseñanza, aprendizaje, ciencia. 


\section{INTRODUCCIÓN}

El presente artículo $^{1}$ se deriva de aprendizajes obtenidos mediante el desarrollo del proyecto de investigación "Concepciones de ciencia, enseñanza y aprendizaje en algunos maestros de ciencias naturales de Bogotá, D.C"2 Reconoce la importancia que presentan las creencias de los profesores para los procesos curriculares, pues la explicitación y análisis de las creencias permiten la estructuración de una visión de mundo desde un paradigma específico en cada uno de los docentes, desde la cual se construye el proceso educativo. A partir del análisis e interpretación de acciones e interacciones de docentes y estudiantes, es posible auscultar e identificar las creencias que mantienen profesores de biología (ciencias naturales) en el grado sexto de la Educación Media Vocacional y Básica. Este trabajo es un aporte al desarrollo de la línea de investigación Acciones de maestros: creencias, roles, metas y contextos de la educación en ciencias, y del grupo Acciones y creencias de maestros de ciencias, formulados dentro de las actividades propias del programa interinstitucional de doctorado en Educación, de la Universidad Pedagógica Nacional.

En la mayoría de nuestras aulas de enseñanza existen problemas comunes que afectan los currículos escolares, problemas sobre los cuales se ha reflexionado poco. Estas reflexiones presuponen fundamentos filosóficos y epistemológicos raras veces presentes en las instituciones educativas. Dado que en Colombia estamos en época de múltiples crisis, este es el momento para identificar oportunidades en la educación de nuestro país, profundizando en el análisis de las creencias de los maestres como parte de la solución de los problemas educativos y proveyendo nuevas visiones de cambio para la educación en ciencias.

\section{ANTECEDENTES}

El estudio de las creencias de los profesores ha estado orientado, en su mayoría, hacia aquellos profesores que se desempeñan en disciplinas de las ciencias naturales. Son realmente escasos los trabajos orientados al estudio de las creencias en profesores de ciencias sociales. En el campo de disciplinas como la física, la química y la biología, ese tipo de investigaciones ha tomado relevancia en las últimas décadas: en efecto, autores como Porlan (1989) y Hodson (1988) han trabajado en esta área, estudiando situaciones problemáticas relacionadas con la enseñanza y el aprendizaje de estas disciplinas.

En el ámbito Internacional. Yerrick, y otros (1997), discuten las creencias que guían las prácticas de los profesores y su relación con el aprendizaje de los estudiantes, encontrando qué las prácticas de enseñanza tradicional no favorecen el aprendizaje de los estudiantes $y$, por el contrario, la forma en que los estudiantes experimentan la instrucción es de gran importancia para desenvolverse de manera exitosa. Olson (1991) y Munby (1983) también reportan como la eficacia de la enseñanza y del aprendizaje estan directamente relacionadas con las creencias que mantienen los profesores acerca de La "ciencia".

El uso de la noción de ciencia en estas investigaciones puede ser entendido de dos maneras. En primer lugar, algunos investigadores, si bien trabajan con profesores que se desempeñan en disciplinas como la física y la biología, tienen un concepto más general

\footnotetext{
${ }^{1}$ Apartes de este texto están contemplados en el informe de investigación presentado a COLCIENCIAS-BID.

${ }^{2}$ Proyecto de Investigación cofinanciado por la UPN-ciun y COLCIENCIAS-BID.
} 
de ciencia, incluyendo entre ellas la amplia gama de posibilidades que ofrecen las disciplinas que abordan el estudio del plexo social (Porlán, 1989). En segundo lugar, algunos investigadores parten del supuesto más restringido según el cual solo son ciencias las disciplinas cuyo objeto es el mundo fisco. La influencia del punto de partida, sobre los resultados de estas investigaciones, es de notarse. Por una parte, quienes parten de un principio mas amplio, pueden reconocer y dar cuenta ce un mayor numero de creencias, pues en sus análisis no desprecian información relativa a criterios propios de las ciencias sociales; por otra, y si lo anterior es cierto, quienes parten de este criterio más amplio, logran mostrar, de manera mas compleja, la influencia que todo tipo de creencias sobre la ciencia ejerce las acciones del maestro. Teniendo en cuenta lo anterior, es pertinente advertir que cuando introducimos el término ciencia en este articulo, no lo restringimos al uso que de el se hace en el ámbito de las disciplinas que abordan como objeto el mundo físico. Por el contrario, cuando hablamos de creencias acerca de la ciencia nos atenemos al uso más general, para dar cuenta de todos los posibles sentidos contenidos en dicha creencia.

Dicho lo anterior, podemos notar como Cronin - Jones (1991) reporta la gran importancia que los profesores asignan al conocimiento de los hechos científicos analizando como muchas de Las creencias de los maestros son incongruentes con las concepciones actuales de la filosofía de Las ciencias y por esto dificultan el desarrollo del currículo. Esta perspectiva podría estar influenciada por una creencia de ciencia un poco menos restringida, pues la filosofía de la ciencia, no lo es solo de las disciplinas cuyo objeto es el mundo natural, o los hechos científicos. Ahora bien, si comparamos este estudio con el realizado por Coben (1989), quien reporta que los profesores nigerianos consideran la ciencia como una forma de producir tecnología útil, visión que según él influye radicalmente en la estructuración de los currículos escolares, podemos notar cómo este último parte del supuesto más restringido de ciencia en el ámbito de la tecnología. Borko y Putman (1996), argumentan cómo las creencias preexistentes influencian el conocimiento del maestro, y al no explicitarlas, analizarlas y criticarlas cuidadosamente se constituyen en limitantes para el cambio.

En el ámbito nacional. En el documento Ciencias Naturales y Educación Ambiental, lineamientos curriculares del Ministerio de Educación Nacional (1998), se hace evidente el clamor por una nueva visión de La educación que posibilite la realización de las potencialidades intelectuales, espirituales, afectivas, éticas y estéticas de los Colombianos, en búsqueda de un ejercicio de convivencia para la paz y la justicia en nuestro medio. Estos planteamientos nos invitan a construir un currículo tendiente a la formación integral y a la reconstrucción de la identidad del ciudadano colombiano.

Ya que en la mayoría de los casos la práctica pedagógica se ha reducido, fundamentalmente, a la enseñanza de contenidos disciplinares (Parra y otros 1994), también se hace referencia a la necesidad de identificar los planteamientos pedagógicos desde los cuales se orienta la acción docente, con el fin de explicitar la identidad del maestro y contribuir a generar mayores posibilidades de solución de problemas (Tamayo 1998). Además, acerca de la disciplina específica, Chona y otros (1998) reportan la forma en que la enseñanza de la biología tiende a ser empírica y especulativa en nuestro medio.

Las anteriores consideraciones de autores nacionales e internacionales nos ponen de presente la relevancia del trabajo en esta área y la necesidad de desarrollar trabajos que, localmente, busquen ampliar los sentidos y reconozcan la importancia de la explicitación y análisis de las creencias de los maestros en el mejoramiento de la educación. 


\section{Políticas nacionales}

En cuanto a las políticas nacionales, la Constitución de 1991 reconoce el acceso al conocimiento, a la ciencia y a la técnica como uno de los bienes y valores de la cultura a la cual tienen derecho los ciudadanos colombianos. La Ley 115 de 1994 o Ley General de Educación, hace referencia a la adquisición de conocimientos científicos y tecnológicos y promueve el acceso de los colombianos a éstos y los demás bienes y valores de la cultura.

La Ley 115 promueve la incorporación de la investigación en los procesos cognoscitivos. Además, plantea la formación de educadores de la más alta calidad científica y ética desarrollando construcciones teóricas y prácticas como eje del conocimiento pedagógico. La Misión de Ciencia y Desarrollo, conformada en 1993, elaboré una serie de documentos que, a partir del análisis de la situación educativa del país, proponían orientaciones acerca del futuro educativo y científico de nuestra patria en el nueve siglo. A pesar de lo anterior, no es claro en el texto de la Ley el concepto de ciencia, pues, en ocasiones parece referirse de manera restringida al mismo, es decir, a las disciplinas cuyo objeto de estudio es el mundo físico exclusivamente, sin reconocer el estudio del plexo social como propio de ciertas formas de hacer ciencia.

Como es conocido, el clima cultural de nuestro país no es muy favorable para el desarrollo de la ciencia y la tecnología, pues se favorecen los resultados inmediatos, prácticos y concretos y poco se favorece el trabajo planeado, a largo plazo y de reflexión profunda. Estos no son obstáculos insalvables y somos precisamente los maestres investigadores, quienes tenemos el reto y la posibilidad de orientar los caminos de la enseñanza de las diversas disciplinas con diferentes visiones de mundo, que conllevan a posibilidades distintas de construcción de futuro en el área de la enseñanza de la ciencia.

\section{REFERENTES CONCEPTUALES}

Desde nuestro punto de vista, las creencias pueden ser consideradas como pioneras de acciones específicas. Muchas de las creencias están tan fuertemente arraigadas que forman parte de nuestra más profunda visión de mundo y actúan como filtros en la auto comprensión de nuestras acciones dando elementos para la compresión de las acciones de los demás.

De acuerdo con Tobin y Lamaster (1995), la creencia es un conocimiento viable, pues posibilite el logro de propósitos particulares en contextos específicos. Estas creencias forman parte de la red de significación del maestro y contribuyen a la estructuración de sus propias redes culturales. Podemos decir que las creencias son base de las acciones del maestro y orientan sus acciones ya sea como practicante, como investigador o como intelectual.

McRobbie y Tobin (1995), consideran que las acciones son de naturaleza cultural y están constituidas, precisamente, por las creencias, los roles, las metas y los contextos en interacción dialéctica. Entonces, se enfatiza la naturaleza cultural de la acción, reconociéndole su componente interpretativo, osca la asignación de significados. En este sentido, la acción está constituida como lo enuncia Erickson (1986): por la conducta física más la ampliación del significado de esa conducta. En consecuencia, entendemos que la acción es intencional, lo cual permite su transformación racional. Una de las preocupaciones centrales de esta investigación constituye el análisis de las creencias que 
acerca de la enseñanza de la biología pose un maestro del grado sexto. Al reconocer que la educación debe ser comprendida como una práctica social de naturaleza histórica y cultural (Carr, 1996), será posible que los profesores de biología puedan ampliar sentidos dentro de la teoría crítica de la educación, constituyéndose ésta como un posible camino de comunicación válida.

Acerca de la enseñanza como acción técnica. Ha consistido principalmente en la transmisión de contenidos disciplinares. El rol del maestro se ha circunscrito a la exposición clara de estos contenidos a sus estudiantes. Los contenidos de la enseñanza se han entendido como resultados formales de procesos científicos, por lo tanto, se aprecian como trascendentales y universalmente válidos. Se considera que algunas habilidades propias del desarrollo del pensamiento científico (en un sentido restringido del término), tales como observar, clasificar, comparar, analizar, predecir y generalizar, pueden dar razón por si solas de hipótesis y teorías científicas. La enseñanza como acción técnica pretende predecir y controlar los resultados de la transmisión de sabores y de los cambios de conducta de los estudiantes, estableciendo claramente los contenidos a enseñar, diseñando las estrategias didácticas adecuadas y evaluando "objetivamente" los resultados obtenidos con los estudiantes.

Acerca de la enseñanza como acción práctica. En este contexto, se concibe que los sujetos históricos son tan diversos como sus propios contenidos de conciencia, lo cual hace necesario que la enseñanza sea vista como un proceso comunicativo, en el cual los sujetos reflexionan acerca de sus propias creencias, las interpretan desde los contextos históricos en los cuales se han desarrollado y buscan establecer consensos sociales. En este caso, el interés técnico da paso al interés práctico que busca la comprensión de las acciones. Al contemplar la enseñanza en el contexto de la producción de acciones culturales, se propician espacios de interacción y comprensión construidos mediante el consenso colectivo de las interacciones sociales del aula. Esta forma de entender la realidad del aula contribuye a ampliar y a mantener la intersubjetivídad entre profesores y estudiantes en búsqueda de consensos que orienten la producción de sabores. Esta dimensión de la enseñanza se orienta hacia una reflexión en la acción o a lo que Schón (1998), ha llamado profesionales "prácticos reflexivos".

Acerca de la enseñanza como acción crítica. Este es un proceso de autorreflexión tanto individual como colectiva, donde se enfatiza en las dimensiones moral, ética y cultural de la enseñanza, reconociendo que ésta es vulnerable a principios políticos e ideológicos. Se rescata la autonomía racional del docente, dándole importancia a la reflexión acerca de la tradición en la construcción del conocimiento y en su naturaleza cultural. Las decisiones y metodologías tomadas son de naturaleza local y provienen de consensos grupales; las relaciones de poder se hacen horizontales promoviendo la autonomía racional y el compromiso social. Por consiguiente, los ambientes de aprendizaje cambian significativamente, lográndose una mayor concientización de la acción. Esta dimensión de la enseñanza tiene como propósito cuestionar creencias acerca de la enseñanza que pueden permanecer inconscientes en los profesionales de la educación y, por tanto, al no ser explicitas, pueden constituirse en barreras que impiden el desarrollo racional de os contenidos educativos. Como resultado, las decisiones tomadas serán más prudentes y mejor informadas pues habrá mayor conciencia en su explicitación. 


\section{LA PROBLEMÁTICA A INVESTIGAR}

Uno de los problemas básicos, de los cuales nos preocupamos en el desarrollo del proyecto de investigación: "Concepciones de ciencia, enseñanza y aprendizaje en algunos maestros de ciencias naturales", es el expresado en a siguiente pregunta: ¿Qué creencias mantienen os profesores de Ciencias Naturales grado $6^{\circ}$, acerca de la naturaleza de la enseñanza de las ciencias? La reflexión sobre la práctica pedagógica, ya sea propia o de pares, se constituye en un ejercicio de producción y ampliación de sentidos. Esta reflexión contribuye a superar las dificultades de la apropiación acrítica de los planteamientos teóricos de las disciplinas y del propio ejercicio docente; además, permite recuperar el papel fundamental de! docente como constructor de conocimiento pedagógico. De esta manera, será el docente quien contribuya a la reformulación de reformas curriculares y criterios de calidad que aporten al mejoramiento de la educación en ciencias.

\section{METODOLOGIA}

La metodología general para el desarrollo de la investigación mencionada, asumió los principios de la investigación cualitativa sugeridos por Guba y Lincoln (1989). Esta estrategia metodológica ofrece a los profesores la oportunidad de reflexionar sobre su propia evolución personal y madurez cognitiva en diferentes dimensiones, no sólo disciplinar sino política y cultural. Además, busca explorar las creencias y acciones de los maestros y enriquecerlas con interpretaciones significativas provenientes de construcciones colectivas.

Esta es una investigación naturalística; es decir, que se realiza in situ. Durante diez meses se asistió de manera continua a los salones de clase, tomando nota y describiendo las acciones e interacciones que se sucedían, orientándonos por la pregunta: ¿Qué es lo que sucede en esta aula de clase?

Algunas de las clases se filmaron para permitir que los docentes, en ocasiones acompañados de sus estudiantes, recordaran y reflexionaran acerca de los planteamientos hechos durante las mismas. Luego se hizo análisis colectivo del contenido.

Un importante punto de apoyo consistió en la trascripción de cada uno de los videos de clase y de las entrevistas realizadas a los docentes. El registro de lo sucedido conté con la consideración de fragmentos cada vez más amplios de la cotidianidad de los profesores. Como en cada caso, los registros de los profesores fueron transcritos y analizados, buscando explicitar formas de pensamiento subyacentes con respecto a la enseñanza de las Ciencias Naturales grado $6^{\circ}$, con la perspectiva de construir nuevos sentidos y de obtener mayor entendimiento e interpretación de las creencias y acciones de estos profesores en el aula.

Otros documentos analizados tuvieron que ver con materiales escritos, elaborados por los maestros y estudiantes, con las evaluaciones realizadas, con los documentos institucionales tales como el Proyecto Educativo Institucional y con el Manual de Convivencia, entre otros. Gracias a la transcripción de este material, fue posible ampliar el análisis de los datos y posibilitar las interpretaciones conjuntas luego de hacer varias lecturas del material. 
Se buscó identificar aspectos culturales, institucionales e individuales que fuesen significativos al momento de hallarle sentido a las acciones de los maestros.

Inicialmente, la entrada a los colegios no fue fácil, pues los maestros, comprensiblemente, anteponían sus vivencias sobre las prácticas investigativas tradicionales, realizadas en las instituciones escolares en las cuales, muchos de ellos, se habían sentido usados. Se recomienda, por lo tanto, emprender cada vez más y con mayor decisión, investigaciones de este carácter, que promuevan cambios culturales de los procesos de investigación en las aulas y creen condiciones confiables y favorables en las que cada vez participen mayor numero de docentes de manera más comprometida.

\section{EL PROCESO DE CAMBIO}

Es importante hacer referencia al temor al cambio que todavía experimentan muchos maestros. Existen dificultades para liderar procesos y el maestro, quiere ir con lo que le es más conocido o con lo que hacen los demás, ya que frecuentemente le ha dado resultados satisfactorios. "Atreverse a innovar es para valientes o para quijotes", enuncia Luis Carlos ${ }^{3}$ en una de las entrevistas. A la mayoría de los maestros, se les dificulta tomar decisiones de manera independiente, y prefieren estar amparados por el anonimato del grupo doblegando de esta manera sus posibilidades de liderazgo y expresión de autonomía. Parece que el docente no posee la fortaleza interna necesaria para cambiar, hecho que refleja la restringida imagen que se tiene del docente en muchos círculos de la sociedad. Por ello, se requiere realizar con mayor frecuencia investigaciones tendientes a auscultar los imaginarios del docente. Es evidente la necesidad de una política nacional que apoye con recursos el deseo de cambio de los maestros.

\section{Perfil de los profesores participantes.}

De acuerdo con el informe de la investigación presentado a Colciencias, a nuestro juicio, algunas características de los profesores en general, y de Ciencias Naturales grado $6^{\circ}$ en particular, que finalmente toman la decisión de participar pueden ser las siguientes:

- Son profesores que han participado en diversos eventos de perfeccionamiento y que mantienen un interés por su actualización permanente.

- Estos profesores, generalmente, son líderes en sus instituciones y toman parte activa en la construcción de propuestas académicas y/o extraescolares.

- Son personas con un alto grado de convicción respecto a la importancia de su saber.

- Generalmente, tienden a trabajar de manera individual y buscan reconocimiento por la labor que desarrollan.

Si bien estas características al parecer posibilitan una toma de posición favorable a participar en la investigación, también constituyen el contexto en el cual aparecen las dificultades que explican la necesidad de una política de Estado que reconozca a los profesores de los colegios como investigadores, en toda su dimensión. Estas

\footnotetext{
${ }^{3}$ Luis Carlos es el nombre que se la asignado como representativo para el maestro sin tener en cuenta el género. Representa una construcción colectiva.
} 
consideraciones son, ante todo, relevantes en el lineamiento de las investigaciones cualitativas, específicamente en los estudios de caso, por cuanto obtener las interpretaciones y los sentidos que los propios docentes atribuyen a sus acciones implica un alto compromiso de su parte, compromiso que exige dedicación de tiempo extra. En este sentido, esta investigación ha sido importante para los investigadores, en tanto que no arrojado claridad acerca de las condiciones, más allá de la buena voluntad y de los buenos propósitos, que este tipo de trabajo requiere.

Perfil de las instituciones participantes. Los estudiantes que atienden los colegios participantes en la investigación son de estrato socioeconómico medio-bajo. Puesto que estas son instituciones de carácter público, a ellas tiene acceso gran parte de la población escolar representativo del estrato social dominante en dichos sectores. En el maestro existe interés por desarrollar autonomía y responsabilidad social en sus educandos, pero es difícil encontrar estas expresiones en la mayoría de los docentes.

\section{CREENCIAS DEL MAESTRO}

Para guiar al lector en la comprensión ce esta parte del texto, es importante anotar que primero se identificó la creencia, luego se extractaron segmentos de clases o entrevistas como evidencia de esta creencia, y a continuación se hizo la interpretación correspondiente.

- Luis Carlos cree que si los estudiantes representan y describen las cosas "tal como son realmente", lograrán un buen aprendizaje.

En el siguiente segmento de clase se pueden evidenciar algunas creencias con respecto a la enseñanza y a la ciencia que maneja el profesor:

Luis Carlos dice:

Hay muchos que preguntaron que sí hacian célula vegetal o animal. Por favor; la célula que quieran, pero tienen que darme todas las características de la célula que eligieron. ¿Si? Voy a nombrar las personas que están con deudas aquí conmigo. Como nuestro trabajo ha sido todo sobre la célula resulta que lo único que he pedido es... la elaboración de la célula.

Buscando que los estudiantes representen en su modelo la célula tal como es, con los organelos que posee y la posible organización de estos organelos dentro de la célula, Luis Carlos enfatiza en la presentación de hechos que en la sustentación de argumentos, mediante los cuales los estudiantes puedan demostrar que han logrado comprensión de la estructura general de una célula. Esta forma de enseñanza es coherente con una visión de ciencia como algo acabado y establecido. Este profesor cree que en ciencias naturales el aprendizaje debe ser concreto. Esto se evidencia en expresiones tales como: boquita cerrada, usted sólo me está explicando ese dibujo, no interesa lo que pase de ahí en adelante, lo único que está hablando es de los centríolos, de la membrana nuclear y de lo que hay dentro del núcleo.

La representación de las cosas "tal como son", evoca una imagen de ciencia rígida, exacta, infalible en la cual las cosas son "tal como son", como en una imagen de espejo, y no pueden ser de otra forma. Estas consideraciones afectan el proceso de enseñanza, pues requieren la comparación y contratación con patrones externos. Bajo este modelo no 
se acepta que el estudiante sea generador de su propio conocimiento, sino que el conocimiento se considera como algo que ya está preelaborado y el estudiante debe acogerse a visiones predeterminadas. El maestro considera que hay mayor rigurosidad si se describen las cosas como son, dudando de la rigurosidad o viabilidad de nuevos modelos que acepten construcciones auténticas de los estudiantes. Le es más fácil volver a prácticas tradicionales sobre las que él considera puede tener control. Por lo demás, se nota una visión de ciencia restringida a las disciplinas cuyo objeto es el mundo físico. Por ello, la enseñanza no puede ser entendida como una acción cultural, cuyo objeto no es la transmisión del conocimiento acerca de ese mundo, sino la formación del sujeto en ámbitos particulares del contexto social, entre los que se cuentan, por supuesto, las disciplinas cuyo objeto es el mundo físico" culturalmente mediado.

- Luis Carlos cree que silos estudiantes cumplen con lo que deben" al profesor. lograrán aprender.

Al respecto. en uno de los apartes de transcripción de los vídeos de este docente se puede extraer lo siguiente:

Voy a nombrar las personas que están con deudas aquí, conmigo. Y hay otros que no tienen lo más elemental, como fue elaborar la célula, la mitosis y hacer una pequeña demostración de lo que aprendieron. Entre esos está: Alberto Gómez ${ }^{4}$, nada de nada. No sé cuál será su laborar aquí en este salón. Me falta Angela Catalina por presentar lo de mitosis y le falta hacer algunas exposiciones porque ya no recibo ese dibujo, y eso no lo recibo si no me dan explicaciones de lo que hicieron en mis clases. Entonces quedan advertidos. Y me da mucha pena, pero ya en esta semana tengo que entregar notas en Coordinación y los ajustes fueron la semana pasada. Rojas Gabriel, también creo que está de visita aquí en el salón, porque no me ha entregado ninguna de las dos cosas y no ha hecho ninguna exposición. Está en deuda aquí conmigo. Me debe lo de la mitosis y la explicación correspondiente (18 de agoste de 1998).

Parece que el maestro considera el conocimiento como algo que fue dado [prestado] por el profesor al estudiante. Por lo tanto, el estudiante "debe" devolver aquello que no es suyo y lo "debe" devolver lo más pronto posible. Es notoria la alusión "me debe", debe". Los estudiantes están en deuda con el profesor, deben" lo de célula.

Al proponer la idea de que es el estudiante quien debe al profesor, se favorece un desplazamiento de los propósitos centrales del aprendizaje y el estudiante asume que debe realizar sus labores para cumplir más con las exigencias de su maestro que para ampliar sus posibilidades de comprensión de fenómenos naturales y gozar con el proceso de aprender.

Además, se asume que es el estudiante quien debe entregar al maestro la asignación requerida y, de esta forma, cumplir con el propósito tácito de que el conocimiento debe ser para entregarlo al maestro para devolvérselo. De esta forma, no se fortalece en el estudiante la concepción de aprender por el deseo de conocer más y de crecer en la construcción de significados que le permitan explicarse a sí mismo el mundo que le rodea. Aún más, al considerar que el estudiante está en deuda con el maestro, el maestro recupera su rol de fuente de conocimiento de donde emana la mayor parte de los conocimientos y donde finalmente deben llegar. En esta situación el maestro es el

\footnotetext{
${ }^{4}$ los nombres han sido cambiados para proteger la privacidad de los participantes.
} 
poseedor de la fortuna del conocimiento. Nos parece que se crea una relación de dependencia donde el estudiante está en desventaja, pues es la persona que debe algo que le ha sido dado y que no es suyo, quien debe devolverlo. Esta situación de dependencia en desigualdad de condiciones no permite el desarrollo de la autonomía y no deja al estudiante en libertad de aprender, pues debe pagar, lo que debe".

\section{Consideraciones con respecto al cambio}

- Luis Carlos cree que tenemos que cambiar para aprender a vivir con la nueva generación.

Al respecto, Luis Carlos comenta:

Por ejemplo, pertenezco al grupo del proyecto de educación sexual y nosotros consideramos y vemos muy claro que para que haya un cambio... , huyyyy!.. pues estamos formados en un tipo totalmente diferente..., en unos patrones de cultura totalmente distintos. La formación de hoy es para las nuevas generaciones.., tenemos que hacer un cambio para aprender a vivir con esta generación porque nosotros tenemos años luz de diferencia con los estudiantes. Además, casi todos los maestros del Distrito ya somos un poquito viejos y si no nos cuestionamos... sí no nos cuestionamos un poquito en la modernidad, pienso que estamos desgastados, de ahí el quejarnos de que tan indisciplinados, de que no nos entienden.., de que no tienen incentivos de trabajo... pero, precisamente es porque ellos hablan.., ven de una manera la vida y nosotros la vemos de otra (Luis Carlos, 24 de Nov.).

Luis Carlos reconoce la gran dificultad que tenemos los maestros para cambiar y poder acercarnos de manera significativa a las nuevas generaciones, pues fuimos formados dentro de unos patrones culturales totalmente diferentes. Podemos observar que el maestro reclama un cambio cultural más que un cambio simplemente de contenidos, reconoce que en el mundo actual ha habido una evolución y que los valores $y$ apreciaciones de las nuevas generaciones ya no son las mismas que cuando se formó, reconoce que hay una brecha entre las dos generaciones: jóvenes y adultos y que la generación de los adultos debe tratar de cerrar esta brecha, acercándose de alguna forma si quiere entender a los jóvenes y trabajar con ellos. La mayoría de maestros del Distrito que participaron en esta investigación tienen bastantes años de experiencia en las aulas y no han tenido muchas oportunidades serias ni permanentes de cuestionar su acción educativa, tampoco han tenido la oportunidad de reconocerse en sus creencias, esta situación dificulta el acercamiento a las nuevas generaciones de estudiantes que van a las instituciones públicas como en nuestro caso especifico.

Es fácil reconocer que los intentos pasados de reformas curriculares, y que los esfuerzos bien intencionados de cambio, no han sido exitosos en mejorar la labor del maestro en las instituciones públicas, ya que estas reformas no han consultado directamente el sistema de creencias del maestro con respecto a los tópicos tratados ni mucho menos con respecto al cambio. La situación continuará siendo la misma sí los investigadores y los administradores no trabajan directamente con los maestros y tratan de entender sus creencias y los problemas a los que ellos se enfrentan día a día. Se necesita reconocer el esfuerzo del maestro al tratar de mejorar sus prácticas y ayudarle en la identificación de sus creencias acerca de la naturaleza de la enseñanza, del aprendizaje y de la ciencia, si queremos que el maestro recupere su autonomía (Reyes Herrera, 1996). 
De acuerdo con Tobin (1993), "un prerrequisito para entender el proceso de cambio es entender la cultura en la cual están embebidos el aprendizaje y la enseñanza”. Por lo tanto, asistir a los maestros a reflexionar en su epistemología de tal forma que puedan entender el aprendizaje, y por ende, la enseñanza de la biología y Ciencias Naturales desde una perspectiva diferente a la habitual, representa un reto y una oportunidad de empowerment en el sentido en que el maestro puede analizar lo que hace en el aula y realizar ajustes que tengan sentido para él. En la medida en que el maestro conozca sus propias creencias y las utilice para hallarle sentido a su quehacer y al de sus estudiantes, la reforma de la enseñanza de la biología y Ciencias Naturales evolucionará. La reflexión del maestro de biología sobre su quehacer contribuye a la interpretación de su propia cultura y a la construcción de su identidad como maestro; esta construcción proviene de negociaciones sociales y de intereses individuales. Por esto, el análisis e interpretación de las acciones e interacciones de los profesores intenta construir identidad cultural a través de la explicitación de las creencias de los docentes y a través del análisis del sistema de valores presente en las relaciones de poder que se evidencian en el aula de clase.

Por lo tanto, la construcción de identidad cultural en los docentes de biología y ciencias naturales a través de la interpretación y análisis de sus creencias, contribuye a la formación del carácter intelectual del maestro. La crítica autónoma de sus creencias le ayudará no sólo a retomar fuerzas para desarrollar al máximo sus posibilidades de enseñar y aprender en la forma que aspira, sino también a controlar su proyecto de vida. Además, consideramos que la identificación de las creencias del docente está a favor de la creación de una educación crítica, autónoma y política que facilitará el crecimiento de la comprensión y que permitirá un genuino control en las esferas de la vida en las cuales participamos no solamente como docentes o como estudiantes sino como ciudadanos comprometidos con el mejoramiento de la enseñanza de la biología y las Ciencias Naturales.

No se trata, como lo dicen Arteta y Reyes (1998), de "cambiar por cambiar, de hacer las cosas de un modo diferente y decir que cambiamos, sino de cambiar para mejorar, y esto implica reflexionar sobre lo que hacemos en las aulas de clase y valorar cada una de las actividades y estrategias que desarrollamos con cada grupo de estudiantes, de tal manera que cuando decidamos cambiar o no cambian" sepamos por qué tomamos esa opción". Además, suele ocurrir que a pesar de que la mayoría de los maestros hablan sobre el cambio y la necesidad de cambiar, no lo pueden hacer, o lo que hacen no es muy efectivo ya que la mayoría no posee los referentes ni las imágenes necesarias para implementar y sostener las anheladas innovaciones (Reyes,1 993).

\section{A MANERA DE CONCLUSIÓN}

Gracias a estos trabajos podemos afirmar que al favorecer la constitución de comunidades de maestros reflexivos, comprometidos con la construcción de conocimiento sobre su quehacer pedagógico, se potencian las posibilidades de cambio en la práctica educativa de los maestros.

Algunas de las reflexiones en cuestión, dejan ver que existe una postura escéptica, generalizada respecto de la enseñanza convencional. Estas reflexiones ofrecen la posibilidad de considerar la ciencia, la enseñanza y el aprendizaje como una acción crítica, develando así el interés emancipatorio que ya subyacía en la ontología tradicional, y que al no cuestionario había permanecido como mito de la educación. En este sentido, el trabajo de revisión biográfica respecto a las investigaciones que se vienen 
desarrollando, tanto en el ámbito nacional como internacional, puede servir como referencia inicial a los profesores interesados en investigar sobre sus propias creencias acerca de la naturaleza de la ciencia, la enseñanza y el aprendizaje.

Hacer explícitas las creencias de los maestros en torno a la ciencia, a la enseñanza y al aprendizaje. y el reflexionar y cuestionarse en forma continua y sistemática sobre ellas, potencia posibilidades de cambio en el ser y quehacer del maestro en pro del mejoramiento de la enseñanza y aprendizaje de la disciplina.

Actualmente, son muy pocos los educadores que reconocen que el cambio sistemático y profundo en la educación se da más en el cambio de las creencias del maestro que en el cambio de las estrategias metodológicas; por esta razón, consideramos que el análisis de las creencias del maestro es fundamental en el proceso de mejoramiento de la enseñanza de las ciencias.

\section{REFERENCIAS BIBLIOGRAFICAS}

Arteta de Molina, J. y Reyes Herrera, L (1998). "La investigación en el aula de ciencias una estrategia para a transformación ce las prácticas de enseñanza ce la Biología". En: Revista de la Asociación Colombiana de Ciencias Biológicas, 10(1-2), 32-38.

Borko, H. y Putnam, R. (1996). Learning to Teach. In D. Berliner \& R. Calfee (eds.), Handbook of Educational Psychology, Macmillan. New York.

Carr, W. (1996). Una teoría para la educación. Hacia una investigación educativa crítica. Madrid: Morata.

Chona, D., G.; Castaño, C., NC.; Arteta, V.; J.; Leudo, A.; M.; Valencia, S. y Martínez, S. (1998). "Aproximación a las creencias que orientan la práctica del profesor de Biología". En: Revista de la Facultad de Ciencia y Tecnología No. 4. Universidad Pedagógica Nacional.

Cobern, W.W. (1989). A comparative analysis of NOSS profiles en Nigerian and American preservice, secondary science teachers. In: Journal of Research la Science Teaching, 26(6), 533-541.

Cronning-Jones, L.L. (1991). Science Teacher beliefs and their influence on curriculum implementation. Two case studies. In: Joumal of Research in Science Teaching. 28(3). 235-250.

Erickson, F. (1986). Qualitative Methods in Hesearch en Teaching. In Merlin C. Wittrock (Ed.). Handbook of Research on Teaching, (3 ${ }^{\text {rd }}$. ed.) 119-161. New York: Macmillan.

Guba, E. G. \& Lincoln, Y.S. (1989). Fourth Generation Evaluation. Newbury Park, CA: Sage.

Hodson D. (1988). Toward a philosophically more and science curriculum. In: School Science Review, 72(1), 19-40.

Ministerio de Educación Nacional. (1998). Ciencias Naturales y Educación Ambiental. Lineamientos Curriculares. Cooperativa editorial Magisterio Bogotá. 
McRobbie, C. \& Tobin, K. (1995). Restraints to reform: The congruence of teacher and students actions a chemistry classroom. In: Journal of Research in Science Teaching, 32(4), 373-385.

Munby. H. (1983). Thirty studies involving the Scientific Attitude inventory: What confidence can we have in this instrument? In: Journal of Research la Science Teaching, $20(21,141-162$.

Olson, M. W. (1991). La investigación acción entra al aula. Argentina: Aíque.

Parra, R., Castañeda, E., Delgadillo, M., Rueda, R., Turriago, O.C. y Vargas. M. (1994). La escuela vacía. Bogotá, Colombia. Tercer Mundo Editores.

Porlan, R. (1989). Teoría del Conocimiento, teoría de la enseñanza y desarrollo profesional: Las concepciones epistemológicas de los profesores. Tesis de Doctorado. Universidad de Sevilla.

Reyes-Herrera, L. (1996). An interpretive study of Social forces that constrain actions and interactions in a science classroom in Colombia. Doctoral dissertation. Florida State University. Tallahassee, Florida.

Reyes-Herrera, L. (1993). Constructivist suggestions to improve science and technology education for all. international Forum en Scientific and Technological Literacy for All. Project 2.000 , UNESCO data base, Paris, France.

Schön. D. (1998). El profesional reflexivo. Cómo piensan los profesionales cuando actúan. Barcelona: Paidós

Tamayo, A. (1998). Cómo identificar formas de enseñanza. Santafé de Bogotá: COLCIENCIAS-UPTC.

Tobin, K. \& Lamaster, S. (1995). Relationships between metaphors, beliefs and actions in a context of science curriculum change. In: Journal of Research in Science Teaching, 32(3) 225-242.

Tobin. K. (1993). Constructivist perspectives on teacher learning. In K. Tobin (Ed.). The practice of constructivism la Science Education. Washington, D.C.: AAA Press.

Yerrick.R.. Parke. L. y Nugent, J. (1997). Struggling to promote deeply rooted change: the "filtering effect" of teachers' beliefs on understanding transformational Views of teaching science. Science Education. 81(2), 137-159. 